\title{
Lactation Consultants' Perceptions of Musculoskeletal Disorders Affecting Breastfeeding
}

A Cross-Sectional Survey

Valérie Lavigne, DC, MSc, IBCLC, RLC ${ }^{1}$

Share this:

Objective: The study aimed to investigate IBCLCs' demographic profile, perceptions of breastfeeding musculoskeletal disorders in infants, and referral patterns.

Method: IBCLCs from Canada and the United States $(N=13,017)$ were invited to participate in an online survey questionnaire emailed by the International Board of Lactation Consultant Examiners.

Results: Response rate was $18.9 \%(\mathrm{~N}=2,457)$. Most IBCLCs had a nursing background (64.8\%) and practiced in a hospital setting (57\%). Most reported referral of infants for musculoskeletal treatment (73.9\%). The first professional referral was made to a pediatrician (47\%), followed by craniosacral therapist (16\%), and chiropractor (14\%). Professional expertise was the chief reason for referral (43\%). The most common latch issues referred for were tongue-tie (27\%), painful latch (24\%), neck problems (18\%), and nonlatching (9\%). Congenital torticollis (25\%) and neck tension (14\%) were the main musculoskeletal problems recognized by IBCLCs. Approximately half the respondents (51\%) were comfortable recognizing musculoskeletal issues in babies. Most respondents (91\%) noticed breastfeeding improvement following manual treatment.

Conclusion: IBCLCs refer to musculoskeletal specialists promptly following the first visit. More collaboration between professions may help foster the wide interprofessional support needed to improve breastfeeding rates.

Keywords: breastfeeding, chiropractic, lactation, torticollis, lactation consultant, manual therapy

Some mothers experiencing breastfeeding difficulties solicit the help of IBCLCs. Well-established protocols for the management of breastfeeding problems can be found in the lactation literature. However, when evaluating the source of dysfunctional nursing, little emphasis is placed on musculoskeletal, biomechanical, and neurological aspects of breastfeeding. IBCLCs are sometimes unable to recognize when latch difficulty is linked to these issues (Hewitt, 1999).

The manual therapy literature points out some common physical findings in infants with breastfeeding difficulties, that is, decreased head rotation, favoring one breast, upper cervical dysfunction, temporomandibular joint (TMJ), and cranial dysfunctions (Bernard \& Alcantara, 2012; Hubbard, 2014; Sheader, 1999; Willis, 2011). Most of the babies in these studies suffered traumatic/difficult births, which may have had an impact on cranial bones, neck and TMJ, and impeded breastfeeding (L. Smith \& Kroeger, 2010; Vallone, 2012). The studies demonstrated that infants were treated

1. valerielavigne@me.com
3-4 times on average using low-force gentle chiropractic manipulation, cranial treatments, and light fingertips treatment (Holleman, Nee, \& Knaap, 2011; Miller, Miller, Sulesund, \& Yevtushenko, 2009; Vallone, 2004). Four studies also reported working in collaboration with lactation consultants (Bernard \& Alcantara, 2012; Hewitt, 1999; Miller et al., 2009; Vallone, 2004). Having the latch evaluated by a professional in addition to chiropractic treatment for musculoskeletal issues showed positive results. Tow and Vallone describe how lactation consultants and chiropractors can collaborate to help mothers successfully breastfeed (Tow \& Vallone, 2009).

In reviewing the lactation literature on the subject, only one article of importance can be found. Wall and Glass (2006) reported a case series of 11 babies with mandibular asymmetry diagnosed as the cause of breastfeeding problems. They concluded that early recognition of lower jaw asymmetry and torticollis was crucial to prevent latch issues and that mothers had success with chiropractic and craniosacral therapy treatment (Wall \& Glass, 2006). This goes to demonstrate a definite lack of literature on the subject within the lactation field. 
There is low-level evidence showing improvement in breastfeeding with manual therapy (Centre for EvidenceBased Medicine, 2009; Gagnier et al., 2013; Green \& Johnson, 2006; Holleman et al., 2011; Miller et al., 2009; Willis, 2011). Therefore, when babies show signs of musculoskeletal issues, IBCLCs may consider referring to a chiropractor or other professional. However, it is uncertain whether IBCLCs are able to recognize when a baby could benefit from a referral. The aims of this study were to investigate IBCLCs' perceptions of musculoskeletal disorders affecting breastfeeding and determine their current practices.

\section{Method}

\section{Sample}

An anonymous online retrospective survey was performed over 32 days between September 11, 2013, and October 11, 2013. IBCLCs from the United States and Canada $(N=13,017)$ were invited to participate via e-mail sent from the International Board of Lactation Consultant Examiners (IBLCE). No incentive was given to participants. The survey was conducted using SurveyMonkey, a web-based survey tool. The survey was open for 1 month, and reminders were sent after 10 and 25 days.

\section{Survey Development and Distribution}

The questionnaire was composed of 17 questions; the first 7 established demographic profiles, and the remaining 10 investigated lactation consultants' practices regarding musculoskeletal issues (Appendix). Twelve lactation consultants were selected by the author to participate in a pilot study and pretesting of the questionnaire. No pretest results were included in the main study.

The descriptive statistics (numbers and percentages) were obtained via "SurveyMonkey" data analysis.

Ethics approval was obtained from the Anglo-European College of Chiropractic Research Ethics Sub-Committee (United Kingdom). The IBLCEs' research committee reviewed the research project prior to initiation.

\section{Results}

The survey yielded a response rate of $18.9 \%(\mathrm{~N}=$ 2,457) over a 32-day period. Most respondents (86\%) were from the United States and 14\% from Canada. The largest number of respondents $(40 \%)$ had been practicing as IBCLCs for less than 5 years, and most of the respondents $(57 \%)$ practiced in a hospital setting. The remainder worked in community clinics (24\%) and private practice (19\%). Most respondents had a nursing background (64.8\%), and the second most common background reported was breastfeeding volunteer/ counselor (12.4\%).

A large proportion of IBCLCs (73.9\%) referred infants with musculoskeletal problems for treatment. Most commonly, they referred to the infant's pediatrician (47\%), followed by craniosacral therapist (16\%), and chiropractor (14\%; Figure 1). Most IBCLCs (43\%) based

\section{Figure 1. Professional referral from IBCLCs for musculoskeletal problems $(N=2,255)$.}

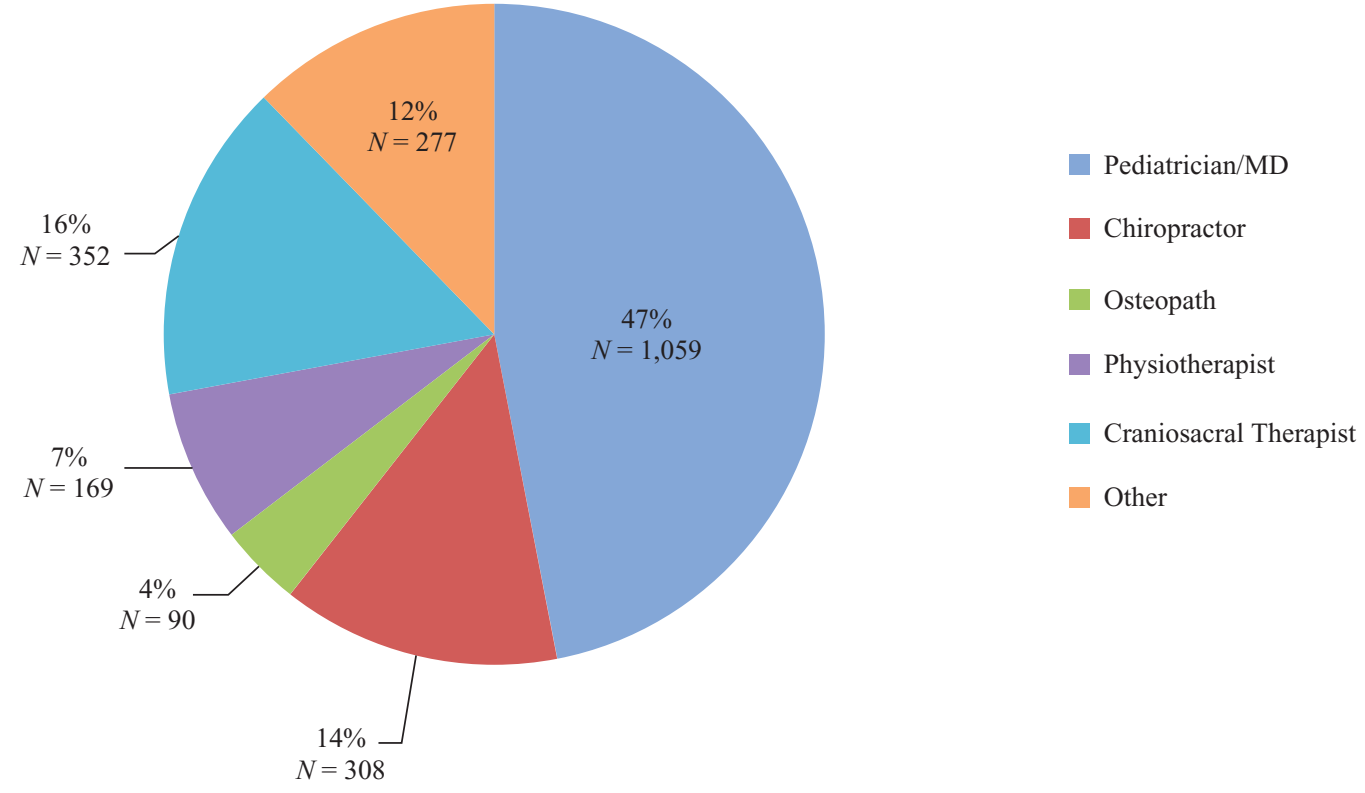


their referral on the professional's level of expertise, followed by reputation (19\%), geographic location (13\%), and a preexisting work relationship with the professional (14\%). Infants recommended for care were $0-3$ weeks old (64\%) and 4-6 weeks old (28\%). The most common latch issue reported and referred for was tongue-tie (27\%), followed by painful latch (24\%), neck problems (18\%), and nonlatching (9\%). Some respondents $(36 \%)$ referred to a professional for an unknown musculoskeletal issue because they were unable to resolve the breastfeeding problem. Congenital torticollis $(25 \%)$ and neck tension (14\%) were the two main musculoskeletal problems recognized by IBCLCs.

Nearly half the respondents (51\%) felt comfortable recognizing musculoskeletal issues in babies, although $41 \%$ were not comfortable at all and only $8 \%$ felt very comfortable. Most referrals were made on the day of the visit (49\%), or within a week (39\%). Most respondents (91\%) noticed an improvement in breastfeeding following treatment by a manual therapy professional.

\section{Discussion}

This survey investigated demographic profile, attitudes, perceptions, and referral patterns of North American IBCLCs to determine practice patterns relative to referral of musculoskeletal dysfunctions detected in the child struggling to breastfeed because these data have never previously been collected. Cook, Heath, and Thompson (2000) reports that an average response rate for an online survey is around 25\%-30\% (Cook et al., 2000). Unfortunately, this survey's lower response rate of $18.9 \%$ will not allow the data to be generalized to the whole population of IBCLCs but is definitely a stepping stone on the subject. This low response rate may be because only IBCLCs interested in the subject decided to participate, which introduced a sample bias. Other limitations associated with survey methodology worth mentioning are recall issues and coverage bias.

First, it was key to establish some parameters for this profession. Generally, IBCLCs come from other professions prior to certification. The literature corroborates the professional backgrounds of IBCLCs described in this survey (Howett \& Lauwers, 2013). This survey also reflected the ratio of the three main work environments shown in other research, that is, hospital, private practice, and community clinic (Shakira, 2011).

An IBCLC is usually sought when breastfeeding is not proceeding successfully. However, they often refer their clients to other professionals when they are unable to solve the problem. Obtaining a second opinion from a peer with more experience has been shown to be common practice in many professions (Barnett, Keating, Christakis, O'Malley, \& Landon, 2012; Forrest, Nutting, Starfield, \& von Schrader, 2002; M. Smith, Greene, Haas, \& Allareddy, 2006). In this survey, IBCLCs first choice of referral, in order of preference, was to a medical doctor, general practitioner or pediatrician, chiropractor, craniosacral therapist, and physiotherapist-all regulated health professions, except for craniosacral therapy.

IBCLCs referred first to health professionals with the most expertise, then best reputation, and finally closest geographic location. Minimizing travel for new parents is important because disruption in routine can negatively impact breastfeeding and decreases the likelihood that parents will follow through with care. Some IBCLCs mentioned that the cost of treatment could prevent parents with minimal means from getting help. IBCLCs may then refer parents to a professional working in a public setting; however, wait time in the public system can be long. The average age of the infant at the time of referral was within the first 3 weeks of life and less often between 4 and 6 weeks. Referral was usually immediately following the first visit to the IBCLCs. These results concur with the critical period of early cessation for breastfeeding (Kronborg \& Væth, 2004; Oakley, Henderson, Redshaw, \& Quigley, 2014).

\section{IBCLC's Comfort With Making Referrals}

In this survey, IBCLCs were asked to rate how comfortable they felt recognizing musculoskeletal problems in infants. Only a small number of IBCLCs reported feeling very comfortable, whereas nearly half did not feel comfortable at all. This could explain why a large percentage of IBCLCs referred to musculoskeletal professionals when they were unable to resolve the breastfeeding or identify musculoskeletal problem. This was an interesting result because it may imply that musculoskeletal issues are not the first thing that comes to mind when IBCLCs are evaluating breastfeeding issues, but more the last thing to try after everything else has failed. However, what if the musculoskeletal problems are actually the root of the issue? By delaying the referral at the end when everything else has been tried, the IBCLCs are delaying the potential improvement in breastfeeding.

The profession may benefit from including training in musculoskeletal problems into its curriculum along with tools to help identify these issues, which may allow IBCLCs to recognize these problems in infants more easily (Table 1). However, IBCLCs reported being able to recognize congenital torticollis and neck tension because these are very common in babies. In this survey, the most 
Table 1. Checklist to Help IBCLCs Identify Musculoskeletal Problems

Question the birth-vacuum or forceps delivery, long pushing phase, and so forth.

Does the baby prefer a side to sleep?

Does the baby prefer a side at the breast?

Does the baby chew at the breast?

Does the baby have tongue-tie? Anterior or posterior?

Does the baby show facial asymmetry? Check ears, eyes, and shape of face or cheeks.

Examine the baby to see if he is able to turn right, left, and go in extensions with no resistance (watch for compensation of the shoulders).

common latch issue that required a referral by IBCLCs was tongue-tie, most probably for a frenotomy evaluation, which has been shown to help with breastfeeding (Berry, Griffiths, \& Westcott, 2012; Buryk, Bloom, \& Shope, 2011; Dollberg, Botzer, Grunis, \& Mimouni, 2006). In the last few years, tongue-ties have been getting more attention from lactation research, and IBCLCs now recognize its impact on breastfeeding (National Institute for Health and Care Excellence, 2005).

Interestingly, some professionals have started to look if there was a link between musculoskeletal issues and tongue-tie. Dr. Vallone explores some of these possible links between tongue-tie and musculoskeletal issues in a chapter of Catherine Watson Genna's book, Supporting Sucking Skills in Breastfeeding Infants (Vallone, 2012). Some of these links discussed are that tongue-tie infants may develop a potential overuse of mastication muscles, submandibular muscles, as they are trying to breastfeed. They may also exhibit a decreased range of motion in the neck as they are trying to suck with a tongue-tie. All these may be derived from the infant's attempts to control the tongue motion while breastfeeding (Vallone, 2012). These issues can be addressed by musculoskeletal treatments to help improve mechanics and potentially have an impact on the tongue motion either before or after the frenotomy has been performed. Other latch issues that required a referral by IBCLCs were painful latch, nonlatching baby, and neck problems.

Most IBCLCs in this survey reported improvement in breastfeeding following musculoskeletal treatments.
Obviously, no conclusion can be drawn from such a subjective question. However, this survey can give a general idea of the high level of satisfaction of IBCLCs with the musculoskeletal treatment to improve breastfeeding. One element to note is that improvement might have also been attributed to the natural history of the problem. Therefore, it is encouraging that IBCLCs in this survey recognize a positive change in breastfeeding following musculoskeletal treatments, and further studies of higher level evidence, such as randomized control trials, could be performed in the future to really assess if musculoskeletal treatments can improve breastfeeding.

\section{Conclusion}

This survey shows that IBCLCs are highly educated professionals who refer most often to musculoskeletal specialists shortly after an initial visit. Although IBCLCs refer to musculoskeletal specialists, they are not always comfortable recognizing infants requiring referrals. Could it be that musculoskeletal treatment is considered a last resort to help breastfeeding after IBCLCs have tried everything? More collaboration between musculoskeletal specialists and IBCLCs may help promote interprofessional support needed to increase breastfeeding rates.

\section{References}

Barnett, M. L., Keating, N. L., Christakis, N. A., O’Malley, A. J., \& Landon, B. E. (2012). Reasons for choice of referral physician among primary care and specialist physicians. Journal of General Internal Medicine, 27(5), 506-512. http://dx.doi.org/10.1007/ s11606-011-1861-z

Bernard, M., \& Alcantara, J. (2012). The chiropractic care of a 6-day-old neonate with breastfeeding difficulties and breastfeeding jaundice. Chiropractic Journal of Australia, 42(3), 108-113.

Berry, J., Griffiths, M., \& Westcott, C. (2012). A double-blind, randomized, controlled trial of tongue-tie division and its immediate effect on breastfeeding. Breastfeeding Medicine, 7, 189-193. http://dx.doi.org/10.1089/bfm.2011.0030

Buryk, M., Bloom, D., \& Shope, T. (2011). Efficacy of neonatal release of ankyloglossia: A randomized trial. Pediatrics, 128(2), 280-288. http://dx.doi.org/10.1542/peds.2011-0077

Centre for Evidence-Based Medicine. (2009). OCEBM levels of evidence. Retrieved from http://www.cebm.net/?o=1025

Cook, C., Heath, F., \& Thompson, R. L. (2000). A meta-analysis of response rates in web or internet-based surveys. Educational and Psychological Measurement, 60(6), 821-836.

Dollberg, S., Botzer, E., Grunis, E., \& Mimouni, F. B. (2006). Immediate nipple pain relief after frenotomy in breast-fed infants with ankyloglossia: A randomized, prospective study. Journal of Pediatric Surgery, 41(9), 1598-1600. 
Forrest, C. B., Nutting, P. A., Starfield, B., \& von Schrader, S. (2002). Family physicians' referral decisions: Results from the ASPN referral study. Journal of Family Practice, 51(3), 215-222.

Gagnier, J. J., Kienle, G., Altman, D. G., Moher, D., Sox, H., \& Riley, D. (2013). The CARE guidelines: Consensus-based clinical case reporting guideline development. Headache, 53(10), 1541-1547. http://dx.doi.org/10.1111/head.12246

Green, B. N., \& Johnson, C. D. (2006). How to write a case report for publication. Journal of Chiropractic Medicine, 5(2), 72-82. http://dx.doi.org/10.1016/s0899-3467(07)60137-2

Hewitt, E. G. (1999). Chiropractic care for infants with dysfunctional nursing: A case series. Journal of Clinical Chiropractic Pediatrics, 4(1), 241-244.

Holleman, A. C., Nee, J., \& Knaap, S. F. (2011). Chiropractic management of breast-feeding difficulties: A case report. Journal of Chiropractic Medicine, 10(3), 199-203. http://dx.doi.org/ 10.1016/j.jcm.2011.01.010

Howett, M., \& Lauwers, J. (2013). The standardization of lactation education to improve professionalism and patient care. Journal of Human Lactation, 29(4), 449-451. http://dx.doi.org/10.1177/ $\underline{0890334413477917}$

Hubbard, M. (2014). Pediatric cholelithiasis and breastfeeding difficulties: A chiropractic case report. Journal of Clinical Chiropractic Pediatrics, 14(2), 1144.

Kronborg, H., \& Væth, M. (2004). The influence of psychosocial factors on the duration of breastfeeding. Scandinavian Journal of Public Health, 32(3), 210-216. http://dx.doi.org/10.1080/ $\underline{14034940310019218}$

Miller, J. E., Miller, L., Sulesund, A. K., \& Yevtushenko, A. (2009). Contribution of chiropractic therapy to resolving suboptimal breastfeeding: A case series of 114 infants. Journal of Manipulative and Physiological Therapeutics, 32(8), 670-674. http://dx.doi.org/ 10.1016/i.jmpt.2009.08.023

National Institute for Health and Care Excellence. (2005). Division of ankyloglossia (tongue-tie) for breastfeeding: NICE interventional procedure guidance [IPG149].

Oakley, L. L., Henderson, J., Redshaw, M., \& Quigley, M. A. (2014). The role of support and other factors in early breastfeeding cessation: An analysis of data from a maternity survey in
England. BMC Pregnancy and Childbirth, 14(1), 88. http://dx.doi .org/10.1186/1471-2393-14-88

Shakira, H. (2011). Position paper on the role and impact of the IBCLC. Morrisville, NC: International Lactation Consultant Association.

Sheader, W. (1999). Chiropractic management of an infant experiencing breastfeeding difficulties and colic: A case study. Journal of Clinical Chiropractic Pediatric, 4(1), 245-247.

Smith, L., \& Kroeger, M. (2010). Cascade of interventions: Physics, forces, and mechanics. In Impact of birthing practices on breastfeeding (2nd. ed., pp. 65-91). Woodhaven, NY: Jones and Bartlett Learning.

Smith, M., Greene, B. R., Haas, M., \& Allareddy, V. (2006). Intra-professional and inter-professional referral patterns of chiropractors. Chiropractic $\mathcal{E}$ Osteopathy, 14, 12. http://dx.doi .org/10.1186/1746-1340-14-12

Tow, J., \& Vallone, S. A. (2009). Development of an integrative relationship in the care of the breastfeeding newborn: Lactation consultant and chiropractor. Journal of Clinical Chiropractic Pediatrics, 10(1), 626-632.

Vallone, S. A. (2004). Chiropractic evaluation and treatment of musculoskeletal dysfunction in infants demonstrating difficulty breastfeeding. Journal of Clinical Chiropractic Pediatrics, 6(1), 349-361.

Vallone, S. A. (2012). Hands in support of breastfeeding: Manual therapy. In K. W. Genna (Ed.), Supporting sucking skills in breastfeeding infants (2nd ed., pp. 271-274). Woodhaven, NY: Jones \& Bartlett Learning.

Wall, V., \& Glass, R. (2006). Mandibular asymmetry and breastfeeding problems: Experience from 11 cases. Journal of Human Lactation, 22(3), 328-334.

Willis, S. A. (2011). The restoration of optimal breastfeeding after chiropractic care in a neonate with breastfeeding difficulties: A case report. Journal of Clinical Chiropractic Pediatrics, 12(1), 873-875.

Acknowledgments: I would like to thank Dr. Joyce Miller for the support, guidance, and help provided during the research; Micheline Winlo, Dr. Di Foglio, and Ghislaine Reid for proofreading the manuscript; and recognize the Goldfarb Breastfeeding Clinic staff for their continuous support and knowledge that inspired this research.

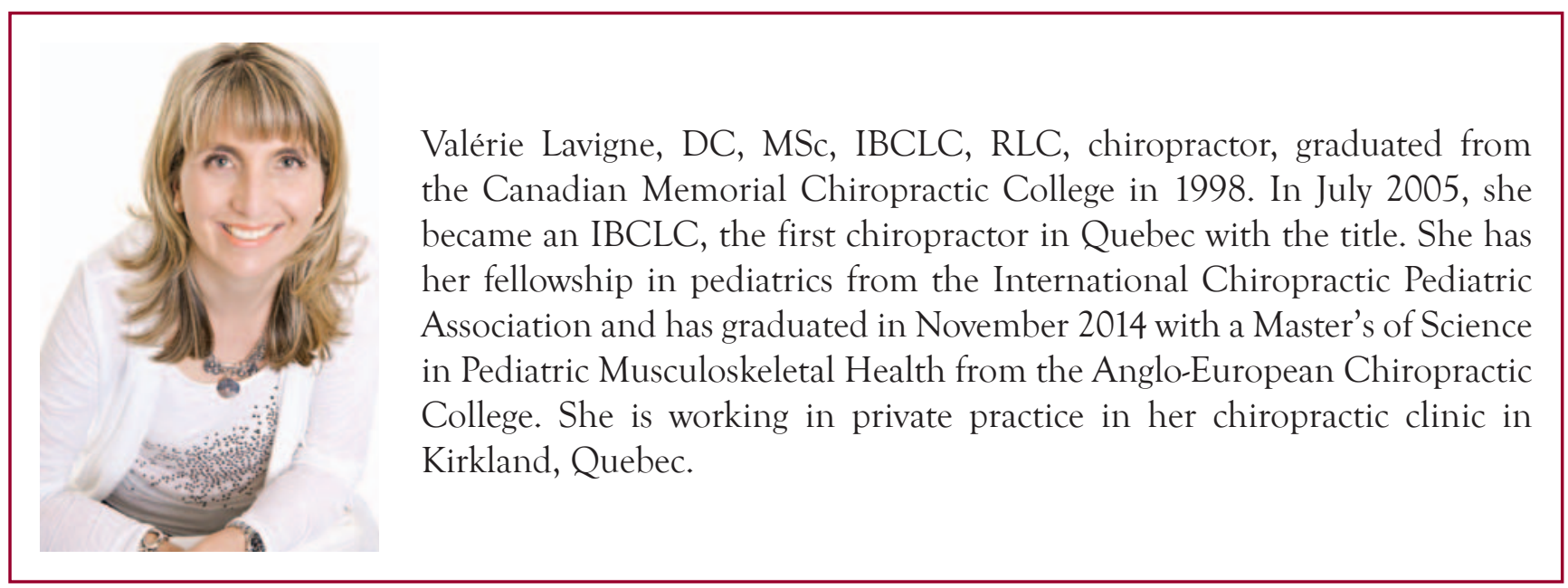




\section{Appendix. Survey Questionnaire}

1. Are you an IBCLC?
A) Yes
B) $\mathrm{No}$
If you answered NO, you may not continue with the survey. Thanks!

2. How many years have you been an IBCLC?
A) $0-5$ years
B) $\quad 6-10$ years
C) $11-15$ years
D) $16-20$ years
E) 21 years plus

3. Where are you from?
A) USA
B) Canada

4. How old are you?
A) 20-29 years old
B) 30-39 years old
C) 40-49 years old
D) 50-59 years old
E) 60 years plus

5. What is your profession/background prior to becoming an IBCLC?
A) Nurse
B) Medical doctor
C) Pharmacist
D) Chiropractor
E) Midwife
F) Breastfeeding volunteer/counselor
G) Other

6. What is your level of education?
A) High school
B) College
C) University
D) Post-graduate

7. Where do you practice?
A) Private practice
B) Hospital based
C) Community clinic

8. Do you recommend patients for musculoskeletal problems such as congenital torticollis, neck tension, and jaw problems?
A) Yes
B) $\mathrm{No}_{\mathrm{o}}$

9. To which professional do you recommend your patients? Please select the main referral.
A) Pediatrician/MD general practitioner
B) Chiropractor
C) Osteopath
D) Physiotherapist
E) Craniosacral therapist
F) Other specify

10. What is the second most common recommendation for your patients?
A) Pediatrician/MD general practitioner
B) Chiropractor
C) Osteopath
D) Physiotherapist
E) Craniosacral therapist
F) Other specify 
11. Why do you recommend this professional? (Choose as many reasons that apply)
A) Level of expertise
B) Geographic location to patient
C) Worked together previously
D) Friend
E) Parent's preference
F) Know by reputation

12. What is the average age of the babies that are recommended for care?
A) 0-3 weeks
B) 3-6 weeks
C) 6-12 weeks
D) 3-6 months
E) Over 6 months

13. What is the most common latch issue you refer for? Please select the main latch issues (more than one answer can apply)
A) Non-latching
B) Painful latch
C) Chewing on the breast
D) Not opening wide
E) Biting
F) Tongue-tie
G) Neck problems

14. What is the most common musculoskeletal issue or discomfort reason you refer for? Please select top two complaints.
A) Congenital torticollis
B) Plagiocephaly
C) Neck tension
D) Chewing on the breast
E) Failure to lie supine (on his back)
F) Unable to resolve breastfeeding difficulty
G) Other specify

15. How comfortable are you at recognizing musculoskeletal issues
A) Not comfortable at all
B) Comfortable
C) Very comfortable

16. How soon after your first visit do you recommend your patients for care?
A) Immediately same day
B) Within 1 week
C) Within 2 weeks
D) Within a month

17. Do you usually see an improvement in the breastfeeding following treatments?
A) Yes
B) $\mathrm{No}_{\mathrm{O}}$

That's the end of the survey. Your responses are appreciated.

Thank you! 